\title{
Thermal aging of polyaniline/grafite nanolayers nanocomposites
}

\author{
Laura Córdova Matte, Cássio Stein Moura, Raiane Valenti Gonçalves, Nara Regina de Souza \\ Basso
}

\begin{abstract}
In this work we investigate the behavior of the electrical conductivity of polyaniline when small amounts of graphite nanolayers are added to the polymeric matrix and subsequently suffer an aging process. We observed that graphite tends to increase conductivity whilst aging tends to decrease it. On the other hand, absorption of atmospheric humidity tends to increase conductivity. We show that a mathematical model describes with good agreement the dependence of the conductivity with the aging time.
\end{abstract}

Index Terms - electrical conductivity; nanomaterials; four probe method; graphite

\section{INTRODUCTION}

Some organic materials can be produced on the form of polymers with high electrical conductivity. There is a strong interest in producing such kind of materials due to their wide range of applications owing to the possibility of customizing their physical and chemical properties1. The synthesis process can be controlled involving the addition of fillers and the combination of various polymers results on unique and desirable characteristics. One example is the polyaniline (Pani) which is a polymer with high electrical conductivity. Pani belongs to the class of intrinsically conducting polymers, which are combined as excellent electrical properties of the metals with a processing flexibility and easy synthesis of the polymers $[2,3]$. Pani is widely studied because of its oxidation-reduction states, which provide its electrical conduction performance. The intermediate oxidation-reduction state of Pani (half oxidized/half reduced) in emeraldine salt form presents higher values of electrical conductivity when compared to the other Pani's oxidation-reduction states $[3,4]$.

Pani nanostructures, such as nanofibers, can be synthesized for the aim of improving the attributes of the material. Polyaniline nanofibers have been studied because they have particular properties that combine the unique characteristics of the convectional polymers with the quantum size effects of nanomaterials [5, 6]. In addition, Pani can be combined with graphite nanolayers (GNL) to produce composite material of excellent electrical behavior [7, 8]. The increase in electrical conductivity of Pani/GNL nanocomposites can be explained

Laura Córdova Matte School of Sciences, Pontifical University of Rio Grande do Sul, Porto Alegre, RS, Brazil., +55 (51) 3320.3545 Cássio Stein Moura, School of Sciences, Pontifical University of Rio Grande do Sul, Porto Alegre, RS, Brazil, +55 (51) 3320.3545

Raiane Valenti Gonçalves Polytechnic School, Pontifical University of Rio Grande do Sul, Porto Alegre, RS, Brazil., +55 (51) 3320.3545

Nara Regina de Souza Basso Polytechnic School, Pontifical University of Rio Grande do Sul, Porto Alegre, RS, Brazil., +55 (51) 3320.3545 by the $\pi-\pi$ interactions between the nanolayers of graphene and the structure of Pani, along with the physical confinement of the Pani molecules in the compact structure of graphene [9, 10]. This condition favors a high degree of ordering of the polymer chain and greater mobility of the charge carriers in the nanocomposite, compared to pure Pani [11]. The combinations of Pani with graphite and its derivatives results in an interesting material with good electrical and electrochemical properties $[12,13]$. All these characteristics makes this nanocomposite a promising material for several applications such as energy storage, capacitors, sensors, solar cells, fuel cells and so on $[12,14,15]$. Capacitors are devices widely used in power electronics applications, i.e., they are submitted to high thermal stress and electrical voltages [16]. Moreover, in other applications, aging acts on the stability of transport properties, being extremely important for charges conduction in polymeric materials. It is therefore crucial to understand the thermal aging behavior of the materials composing such kind of devices [17].

During the thermal treatment of Pani, several chemical processes happen leading to the loss of its electrical conductivity, e. g., decrease on the amount on the dopant concentration and oxidation of the polymeric chain [18]. The rate at which the dopant loss takes place depends not only on the temperature but on the dopant type and the material's morphology. Typically, the high Pani conductivity can be associated to the existence of conducting grains immersed in an insulating matrix [19]. Thus, the overall effect of such events that lead to the thermal aging process is the decrease on the size of the conducting grains. The evolution of the polymer conductivity as a function of treatment time $\sigma(\mathrm{t})$ is given by the following equation [20,21, 22].

$$
\sigma(t)=\sigma_{0} e^{-\sqrt{\frac{E}{\tau}}}
$$

This equation indicates an exponential decrease from the initial conductivity $\sigma_{0}$ as the treatment time increases. The decrease rate depends intrinsically on the material and is described by the characteristic time $\tau$. This parameter can be used to compare the time stability of different substances.

Cabezas et al. [17] reported the effects of the thermal aging on films formed by polyaniline and carbon nanotubes. The addition of the graphene-derived material delays the loss of the Pani dopants, which postpone the degradation of the conductivity of the material. Upon prolonged heating, the nanocomposite film presented semiconductor electrical behavior, whist the pure Pani film exhibited insulating behavior. 
In the current study, we measured the electrical characteristics of samples of Pani and its time evolution during a thermal aging process. In order to foresee the material useful lifespan and practical applications it is mandatory to know its electrical stability. Electrical conductivity was measured using the four probe method. Thermal aging was performed keeping the samples during a preset time on a temperature close but bellow the material's melting point. Samples of pure Pani and containing different amounts of graphite nanolayers were tested.

\section{EXPERIMENTAL}

\section{A. Materials}

Aniline (Sigma-Aldrich) was purified by distillation before polymerization. Natural graphite (Sigma-Aldrich), ammonium persulfate (Sigma-Aldrich) and hydrochloric acid (Merck) were used as received.

\section{B. Synthesis of graphite nanolayers (GNL)}

A graphite structure was modified to graphite nanolayers according to the methodology described elsewhere [23, 24]. Natural graphite in flake form (2.0 g) was chemically treated with sulfuric acid $(24.0 \mathrm{~mL})$ and nitric acid $(6.0 \mathrm{~mL})$ under magnetic stirring for 24 hours. After dispersion, the product was washed with distilled water until becoming neutralized and was dried at $100{ }^{\circ} \mathrm{C}$ for 3 hours. Then, the intercalated graphite was obtained and was heated in air at $1000{ }^{\circ} \mathrm{C}$ for 30 seconds to obtain the expanded graphite. Graphite nanolayers were obtained from the expanded graphite; they were treated with an ultrasound bath Unique (Ultracleaner 1600A, $40 \mathrm{kHz}$ ) in aqueous alcohol solution (70\%) during 8 hours.

\section{Synthesis and characterization of the nanocomposites polyaniline/graphite nanolayers}

Doped Pani was synthesized in the presence of graphite nanolayers by rapid-mixing polymerization using hydrochloric acid as dopant and ammonium persulfate (APS) as oxidant, according to the process adapted from the literature $[25,26]$. In a typical polymerization reaction, GNL ( 5 and $20 \mathrm{wt} \%$ relative to the weight of aniline) and aniline $(0.6 \mathrm{~mL})$ were dispersed in 1.0 $\mathrm{M}$ hydrochloric acid aqueous solution $(20 \mathrm{~mL})$. The dispersion was treated with an ultrasound bath during 1 hour. Then, 1.0 $\mathrm{M}$ hydrochloric acid aqueous solution $(20 \mathrm{~mL})$ containing APS $(0.38 \mathrm{~g})$ was rapidly mixed to the $\mathrm{GNL} /$ aniline/ $\mathrm{HCl}$ dispersion. The reaction occurred at $60{ }^{\circ} \mathrm{C}$ without stirring for 2 hours. The product was then filtered, washed with pure acetone to remove oligomers and dried for $48 \mathrm{~h}$ at room temperature in a desiccator. This methodology in the absence of GNL was also used to produce pure Pani.

The morphological characterization of the samples was performed by scanning electron microscopy (SEM) with a Philips microscope, model XL30, operating at $20 \mathrm{kV}$. The samples were prepared by deposition in an aluminum stub and posterior gold metalization. The diameters of the Pani grains as well as the thickness of GNL were measured by the ImageJ software.

\section{Thermal aging analysis}

In the four-probe method, the collinear electrodes are placed in contact with the sample's surface and a voltage is applied to two of them. In the other two electrodes the electrical current is measured. This method is superior to the two probe method for there is no need to consider the effects of contact resistance neither the cable resistance. We used a system provided by Lucas-Signatore, Model S-302-4 with gold electrodes. Voltages from 1 up to $5 \mathrm{~V}$ were applied (Icel Manaus, PS-6000) resulting in currents of the order of 30 to $500 \mu \mathrm{A}$. Voltage and current were measured with the following multimeters, respectively: Minipa (ET-2510) and Icel Manaus (MD-6700). Plots of voltage versus current allowed through the first Ohm's law the values of the sample's resistance. The second Ohm's law yields the material's resistivity. In order to take into account the finite size of the sample, geometric factors were used [27, 28]. For each sample, the measurement was repeated at least thrice and arithmetic averages were considered. Calibration of the experimental setup was performed with samples of silicon with known resistivity. The silicon standards had nominal resistivities in the range of 1 to $20 \Omega . \mathrm{cm}$. After repetition of 30 measurements, we obtained the value of $5.3 \pm 0.6 \Omega . \mathrm{cm}$, showing good agreement to the expected values.

One sample of pure Pani and two samples of Pani containing 5 and $20 \%$ each of graphite nanolayers (GNL) were treated at $100^{\circ} \mathrm{C}$ in an electrical furnace (DeLeo). Initial treatment time varied from 15 to 60 minutes. Longer treatments steps reached 200 minutes. Afterward, the samples rested in room temperature exposed to air during 170 days in order to infer the atmospheric effect on the samples. After the resting period, a new cycle of thermal treatment was performed in order to accelerate aging. Conductivity was measured at room temperature in between each thermal treatment stage.

\section{RESULTS AND DISCUSSION}

The morphology of pure Pani, GNL and Pani/GNL nanocomposite are presented in Fig. 1. Pure Pani nanofibers were produced by rapid-mixing polymerization, Fig. 1(a). It is observed that well-defined pure Pani nanofibers with an average diameter of $56 \pm 14 \mathrm{~nm}$ were obtained, without the presence of agglomerates. This morphology can be related to rapid-mixing polymerization. This methodology can control the secondary growth of Pani, avoiding the growth of irregular structures, i.e., agglomerates [25]. As can be seen in Fig. 1(b), GNL were successfully obtained through thermal reduction of graphite oxide. It presented stacked graphene layers, which have a thickness of approximately $95 \pm 25 \mathrm{~nm}$, Fig. 1(c). Pani nanofibers were prepared in the presence of GNL, because when the aniline monomer is added to the GNL suspension, the aniline immediately adheres to the surface of the graphene due to electrostatic interactions. During the polymerization stage, GNL provides a high number of active sites for the nucleation of Pani, acting as a support material [29]. Thus, from Fig. 1 (d) it is possible to visualize the growth of well dispersed Pani nanofibers on the surfaces of GNL. The uniform dispersion of polyaniline on the lamellar surface of GNL favors the formation of an efficient network for the charge transport. The $\pi$ bonds present on the GNL surface may interact strongly with the quinoid structures of Pani. This interaction facilitates the process of charge transfer between the components of the system and increases the efficiency of displacement of electrons, thus increasing the electrical conductivity of the hybrid material $[29,30]$. 

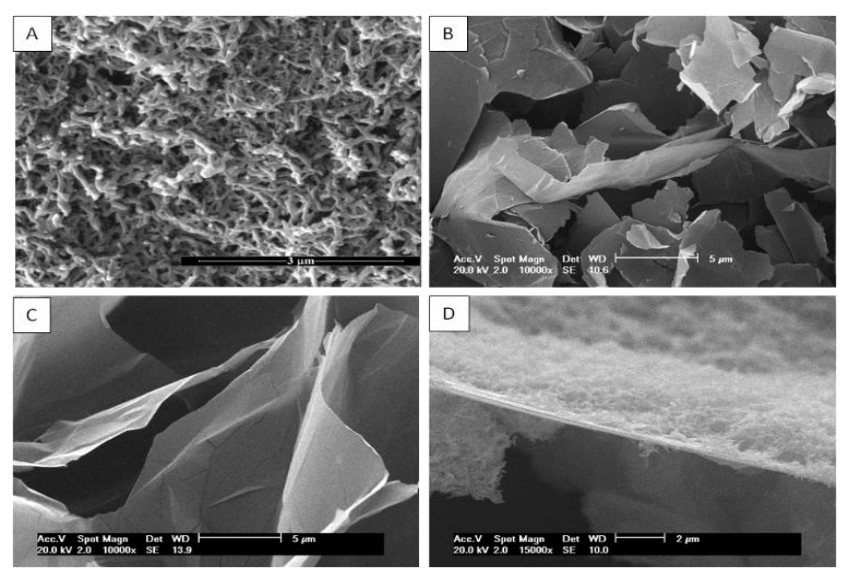

Fig. 1 - SEM images of (a) Pani pure, (b,c) GNL and (d) Pani/GNL nanocomposite.

The conductivity decrease due to the thermal aging is shown in Fig. 2 to 4 for pure Pani, Pani with $5 \%$ of graphite nanolayers and Pani with $20 \%$ of graphite nanolayers, respectively. The dots represent the experimental values. Error bars were estimated through three measurements of the resistivity on each sample. The solid line represents the best numerical fit of equation (1) to the data during the whole time span. One can observe that there is a good agreement between the experiment and the theoretical model for the thermal aging of the materials either for the pure samples as well as for those with GNL, indicating that the processes that occur during the aging of the polymer are mainly responsible for conductivity decrease in all cases. It can be observed that there is a rapid decrease on samples' conductivity as the aging time increases. For very long times the conductivity decreases very slowly indicating a stabilization of the electrical properties.

The parameters used to fit equation (1) to the experimental data are shown on Table 1. As one can see on the middle column, the conductivity increases as the amount of graphite goes up as expected, for graphite is a better conductor than the polymer. The third column shows the characteristic time of each material. The analytical interpretation of equation (1) shows that the larger the $\tau$ is the longer the material remains closer to the initial value. In other words, larger values of the characteristic time provide longer lasting materials. Therefore, comparing the three materials, pure Pani is the least stable among them. The addition of GNL, besides increasing electrical conductivity, increases the material endurance. Nonetheless, when the GNL concentration goes up from 5 to $20 \%$, the characteristic time suffers a decrease showing that there is an optimal value for the capability to avoid aging.

TABLE

Table 1 - Numerical parameters used to fit the experimental data.

\begin{tabular}{ccc}
\hline Sample & $\sigma_{0}(\mathrm{~S} / \mathrm{cm})$ & $\tau\left(\mathrm{min}^{-1}\right)$ \\
\hline Pure Pani & 0.22 & 251 \\
\hline Pani + 5\% GNL & 0.26 & 654 \\
\hline Pani + 20\% GNL & 0.46 & 473 \\
\hline
\end{tabular}

Fig. 2 - Time evolution of the electrical conductivity of pure polyaniline. The fitted curve has a decaying time of 251 $\min ^{-1}$.

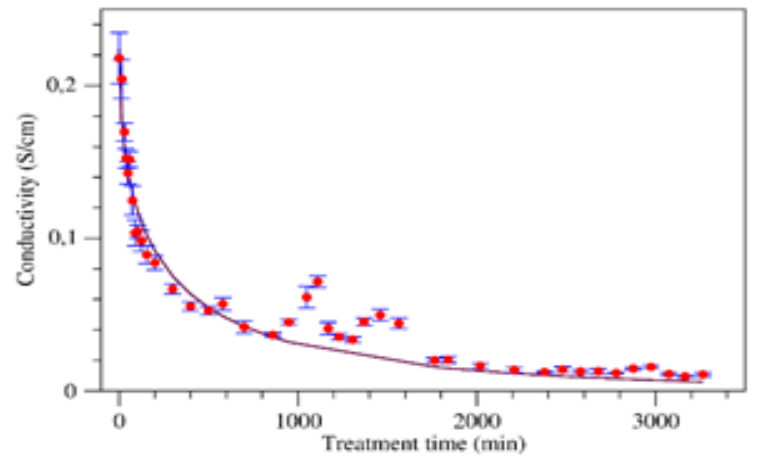

Fig. 3 - Time evolution of the electrical conductivity of polyaniline containing 5\% GNL. The fitted curve has a decaying time of $654 \mathrm{~min}^{-1}$.

In the middle region of the time span there is a noticeable discrepancy between the experimental data and the theoretical model given by equation (1). These values correspond to the 170 days interval when the samples were let to rest in air. We believe that this fact was due to different resting periods between aging steps, which is allowed by different exposure time to atmospheric water. Pani is known for its hygroscopic characteristic, and water tends to increase the samples' conductivity, through the enlargement of the effective size of conducting grains [31]. The thermal treatment applied after the resting time followed the previous protocol, e. g., heating during 15 to 60 minutes stages. It took a few hundreds of minutes to vaporize enough water in order to the samples' conductivity fall again on top of the theoretical curve.

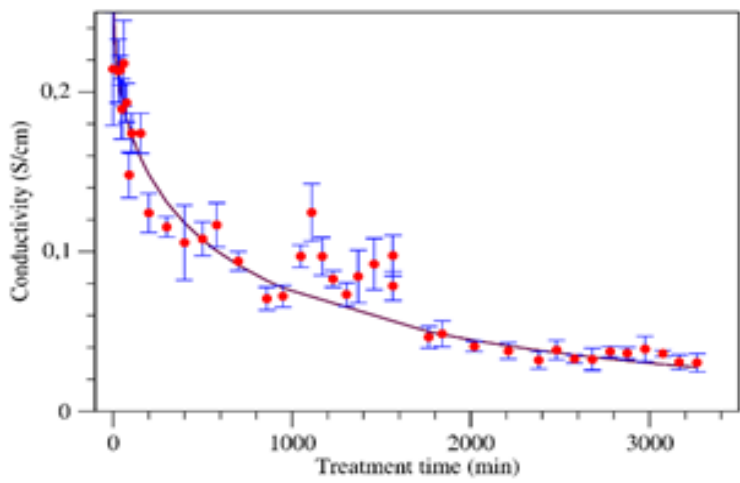

Fig. 4 - Time evolution of the electrical conductivity of polyaniline containing $20 \%$ GNL. The fitted curve has a decaying time of $473 \mathrm{~min}^{-1}$.

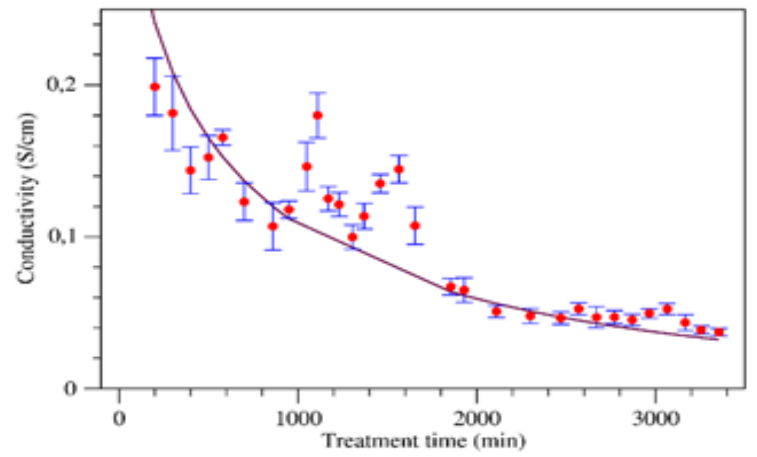

Fig. 5 - Time evolution of the normalized conductivity of polyaniline samples containing different amounts of GNL and exposed to air. The lines are only to guide the eyes. 
In order to clarify the atmospheric effect on each sample we followed its conductivity right after being taken off the furnace until a 5 day resting period. Fig. 5 shows that the conductivity increases for all samples after being exposed to air. We have chosen to normalize all three conductivities to the initial value right after leaving the furnace in order to be able to compare them to each other. Fig. 6 shows the normalized mass after taking the samples out of the furnace. A clear mass increase is observable. Recalling the hygroscopic behavior of Pani it is reasonable to suppose that during the air exposition the samples absorb water and increase their electrical conductivity. Similar results have already been reported [31].

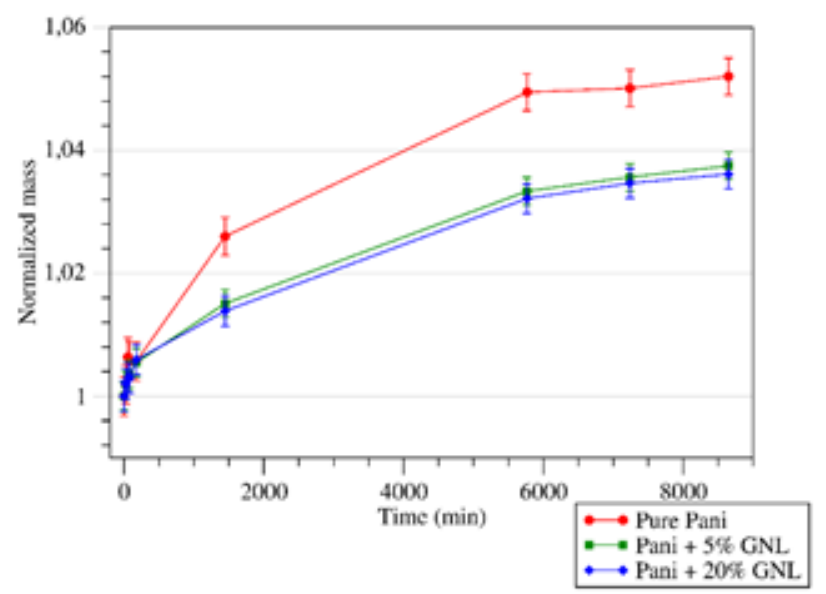

Fig. 6 - Time evolution of the mass of polyaniline samples containing different amounts of GNL and exposed to air. The lines are only to guide the eyes.

\section{CONCLUSION}

In this work we have studied the electrical response of Pani samples to thermal aging and the addition of nanofillers. We observed that the electrical conductivity decreases in a fast way for short treating times and reaches an almost stable state after long periods of aging. A mathematical model describes reasonably the conductivity behavior showing an exponential type of decrease. We have added graphite nanolayers to the polymeric matrix and observed that they improve conductivity and endurance. The same equation can be used to model the aging process of Pani samples with and without graphite nanolayers. Due to the fact that Pani is hygroscopic an increase on the conductivity is observed when samples are exposed to atmospheric air.

\section{AKNOLEDGEMET}

The authors acknowledge the Brazilian Council for Scientific and Technological Development for the financial support..

\section{REFERENCES}

[1] G. O. Young, "Synthetic structure of industrial plastics (Book style with paper title and editor)," in Plastics, 2nd ed. vol. 3, J. Peters, Ed. New York: McGraw-Hill, 1964, pp. 15-64.

[2] W.-K. Chen, Linear Networks and Systems (Book style). Belmont, CA: Wadsworth, 1993, pp. 123-135.

[3] H. Poor, An Introduction to Signal Detection and Estimation. New York: Springer-Verlag, 1985, ch. 4.

[4] B. Smith, "An approach to graphs of linear forms (Unpublished work style)," unpublished.
[5] E. H. Miller, "A note on reflector arrays (Periodical style-Accepted for publication)," IEEE Trans. Antennas Propagat., to be published.

[6] J. Wang, "Fundamentals of erbium-doped fiber amplifiers arrays (Periodical style-Submitted for publication)," IEEE J. Quantum Electron., submitted for publication.

[7] C. J. Kaufman, Rocky Mountain Research Lab., Boulder, CO, private communication, May 1995.

[8] Y. Yorozu, M. Hirano, K. Oka, and Y. Tagawa, "Electron spectroscopy studies on magneto-optical media and plastic substrate interfaces(Translation Journals style)," IEEE Transl. J. Magn.Jpn., vol. 2, Aug. 1987, pp. 740-741 [Dig. $9^{\text {th }}$ Annu. Conf. Magnetics Japan, 1982, p. 301].

[9] M. Young, The Techincal Writers Handbook. Mill Valley, CA University Science, 1989.

[10] (Basic Book/Monograph Online Sources) J. K. Author. (year, month, day). Title (edition) [Type of medium]. Volume(issue). Available: http://www.(URL)

[11] J. Jones. (1991, May 10). Networks (2nd ed.) [Online]. Available: http://www.atm.com

[12] (Journal Online Sources style) K. Author. (year, month). Title. Journal [Type of medium]. Volume(issue), paging if given. Available: http://www.(URL)

[13] R. J. Vidmar. (1992, August). On the use of atmospheric plasmas as electromagnetic reflectors. IEEE Trans. Plasma Sci. [Online]. 21(3). pp. 876-880. Available: http://www.halcyon.com/pub/journals/21ps03-vidmar

[14] K. M.Ziadan. Conducting polymers application. [S.1.]: INTECH Open Access Publisher, 2012.

[15] R. V. Gonçalves, M. L. Zanini, J. A. Malmonge, L. Bonnaud, N. R S. Basso. Pristine cardanol as biobased dopant for polyaniline. Materials Letters. 2016;185:327-330.

[16] W. S. Fagundes, F. F. S. Xavier, L. K. Santana, M. E. Azevedo, S. C. Canobre, F. A. Amaral. PAni-coated LiFePO4 Synthesized by a Low Temperature Solvothermal Method. Materials Research. 2019;22(1):e20180566.

[17] Y. Matsuura. Tunnel magnetoresistance in polyaniline. Synthetic Metals. 2018;243:90-96.

[18] R. V. Gonçalves, M. L. Zanini, J. A. Malmonge, L. Bonnaud, N. R. S. Basso NRS. Cashew nut shell liquid, a valuable raw material for generating semiconductive polyaniline nanofibers. Polímeros: Ciência e Tecnologia. 2018;28:61-68.

[19] M. Oyharçabal, T. Olinga, M. Foulc, V. Vigneras. Polyaniline/clay as nanostructured conductive filler for electrically conductive epoxy composites. Influence of filler morphology, chemical, nature of reagents, and curing conditions on composite conductivity. Synthetic Metals. 2012;162:555-562

[20] A. M. Lentz, G. Gheno, T. Maraschin, J. A. Malmonge, N. R. S. Basso, N. M. Balzaretti, et al. Nanocomposites of polyethylene/polyaniline/graphite with special morphology. Polymer Composites. 2018;39(10):3645-3655.

[21] G. Gheno, N. R. S. Basso, R. Hubler. Polyaniline/Graphite Nanocomposites: Synthesis and Characterization. Macromolecular Symposia. 2011;299-300:74-80.

[22] P. Saha, D. K. Pyne, P. Dutta, A. Halder. Tunable luminescence of graphene oxide-polyaniline nano-composite: Effect of an anionic surfactant. Journal of Luminescence. 2019;206:218-226.

[23] X. Xi, R. Liu, T. Huang, Y. Xu, D Wu. Strong coupled polyaniline/graphene hybrids with much enhanced capacitance performance. Journal of Colloid and Interface Science. 2016;483:34-40

[24] M. O. Ansari, N. M. Khan, S. A. Ansari, I. Amal, J. Lee, M. H. Cho pTSA doped conducting graphene/polyaniline nanocomposite fibers: Thermoelectric behavior and electrode analysis. Chemical Engineering Journal. 2014;242:155-161.

[25] A. Ladrón-de-Guevara, A. Boscá, J. Pedrós, E. Climent-Pascual, A. Andrés, F. Calle, J. Martínez. Reduced graphene oxide/polyaniline electrochemical supercapacitors fabricated by laser. Applied Surface Science. 2019;467-468:691-697.

[26] S. Wang, K. Zhang, Q. Wang, Y. Fan, J. Shen, L. Li, et al. Graphene quantum dot-assisted preparation of water-borne reduced graphene oxide/polyaniline: From composite powder to layer-by-layer self-assembly film and performance enhancement. Electrochimica Acta. 2019;295:29-38.

[27] J. Li, D. Xiao, Y. Ren, H. Liu, Z. Chen, J. Xiao. Bridging of adjacent graphene/polyaniline layers with polyaniline nanofibers for supercapacitor electrode materials. Electrochimica Acta 2019;300:193-201. 
[28] M. Anju, N. K. Renuka. Graphene-dye hybrid optical sensors. Nano-Structures \& Nano-Objects. 2019;17:194-217.

[29] M. Makdessi, A. Sari, P. Venet. Metallized Polymer film capacitors ageing law based on capacitance degradation. Microelectronics Reliability. 2014;54:1823-1827.

[30] A. L. Cabezas, Y. Feng, L. Zheng, Z. Zhang. Thermal ageing of electrical conductivity in carbon nanotube/polyaniline composite films. Carbon. 2013;59:270-277.

[31] A. Wolter, P. Rannou, J. P. Traverset. Model for aging in hcl-protonated polyaniline: structure, conductivity, and composition studies. Physical Review B. 1998;58(12):7637-7647.

[32] D. Jeon, J. Kim, M. C. Gallagher, R. F. Willis. Scanning tunneling spectroscopic evidence for granular metallic conductivity in conducting polymeric polyaniline. Science. 1992;256(5064):1662-1664.

[33] I. Šedenková, J. Prokeš, M. Trchová, J. Stejskal. Conformational transition in polyaniline films-spectroscopic and conductivity studies of ageing. Polymer Degradation and Stability. 2008;93(2):428-435.

[34] H. Qiu, W. Wu, J. Li , K. Fang, W. Mao . Effect of vacuum annealing on characteristics of the hcl-doped polyaniline pellets. Synthetic metals. 2005;155(3):560-564.

[35] S. Sakkopoulos, E. Vitoratos, E. Dalas. Conductivity degradation due to thermal aging in conducting polyaniline and polypyrrole. Synthetic metals. 1998;92(1): 63-67.

[36] F. C. Fim, J. M. Guterres, N. R. S. Basso, G. B. Galland. Polyethylene/Graphite Nanocomposites Obtained by In Situ Polymerization. Journal of Polymer Science: Part A: Polymer Chemistry. 2010;48:692-698.

[37] G. Pavoski, T. Maraschin, F. C. Fim, N. M. Balzaretti, G. N. Galland, C. S. Moura, N. R. S. Basso. Few Layer Reduced Graphene Oxide: Evaluation of the Best Experimental Conditions for Easy Production. Materials Research. 2017;20(1):53-61.

[38] J. Deng, J. Guo, P. Liu. Growth of polyaniline nanomaterials in rapid-mixing polymerization. Colloids and Surfaces A: Physicochemical Engineering Aspects. 2017;521:247-250.

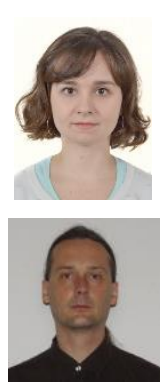

Laura Córdova Matte graduated on Philosophy and Law at UFRGS, and Physics at PUCRS. Earned a master's degree at UFRGS in 2019. Has experience in the area of Physics. Focused, mainly, in the subjects: nanocomposites, spin coating, crystallinity degree.

Cássio Stein Moura, Matte graduated on Physics at PUCRS. Earned the PhD at UFRGS in 2002. Has experience in several areas of Physics: magnetic materials, geophysics, molecular dynamics, nanocomposites, and experimental Physics.

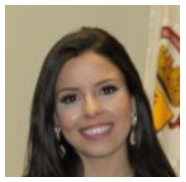

Raiane Valenti Gonçalves graduated on Chemical Engineer at PUCRS. Earned a PhD degree at PUCRS in 2019. Has experience in the area Chemistry Focused, mainly, in the subjects: nanocomposites, polymeric materials, extraction of essential oil and simulation of turbidity currents.

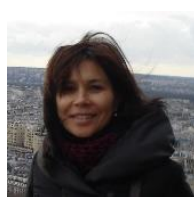

Nara Regina de Souza Basso1 graduated on Chemistry at PUCRS. Earned a PhD degree at UFRGS in 1991. Has experience in the area Chemistry Focused, mainly, in the subjects:, polymeric materials, synthesis and applications. 